\title{
Implementation the Technique of Orthogonal Frequency Division Multiplexing Using 16-Point Fast Fourier Transform and Inverse Fast Fourier Transform
}

\author{
Muhammad Waqas, Muhammad Junaid Khan, Muhammad Abid Saeed \\ Muhammad Sheraz, Muhammad Tayyab Qaiser \\ _DepartmentofElectricalEngineering,SarhadUniversityofScienceand InformationTechnology,Peshawar \\ Pakistan \\ -DepartmentofCommunicationEngineering,UniversityofOulu,Oulu,Finland \\ DepartmentofElectricalEngineering,COMSATSUniversity,Abbottabad,Pakistan
}

\begin{abstract}
Orthogonal FrequencyDivisionMultiplexing(OFDM)isamulti-carriermodulationtechniquewhich dividestheavailable spectrumintomanycarriers.OFDMusesthespectrumefficiently comparedtoFDMAby spacing the channels much closer together and making all carriers orthogonal to one another to prevent interferencebetweenthecloselyspacedcarriers. ThemainadvantageofOFDMistheirrobustnesstochannel

fadinginwirelessenvironment. TheobjectiveofthisworkistodesignandimplementanOFDMtransmitter and receiverusingMATLAB.Theworkconcentrates ondevelopingFastFourierTransform(FFT)andInverseFast FourierTransform(IFFT).The workalsoincludesindesigninga mappingmodule,serialtoparallelandparallelto serialconvertermodule.Thedesignuses16-pointsFFTandIFFTfortheprocessingmodulewhichindicatesthat theprocessingblockcontain16inputsdata.AllmodulesareimplementingusingMATLAB.

Keywords: Orthogonal FrequencyDivisionMultiplexing(OFDM),FastFourierTransform(FFT),InverseFast FourierTransform(IFFT),CyclicPrefix(CP),Quadrature AmplitudeModulation (QAM),Quadrature Amplitude Demodulation(QAD),Encoder,Decoder
\end{abstract}

\section{Introduction}

Theincreasingroleofmultimediaandcomputerapplicationsincommunications hasintensifiedthe researchinthefieldofWirelessBroadbandMultimediaCommunication Systems(WBMCS).Broadbandsystems refertosystemswithveryhigh datarates.Overthelastfewyears,Ultra-Wideband(UWB) communicationsystems havegainedsignificantinterestfrom industry[1]-[2].Thereasonbehindthisisthatthetechnology promisesto deliververyhighdataratesranging from 110Mbpsatadistanceof10metersupto480Mbpsatadistanceof 2 metersina real multipathchannel,whileconsumingverylittlepoweras wellas havinga small sizeandinsignificant weight.Thetransmission ofsuchhighdataratesoverrealchannelsinamultipath environmentimposeslarge bandwidths, thuspushingcarrierfrequenciestoveryhighlevels [3]. Transmittingsuch highdata ratesthat canresist allradiochannelimpairments requiresacarefulchoiceofmodulation technique.OrthogonalFrequencyDivision Multiplexing(OFDM)seemstobea verygoodchoice.OFDMisknownforitshighspectralefficiency,italsooffers inherentresistancetonarrowbandinterference.OrthogonalFrequencyDivisionMultiplexing(OFDM)multicarrier modulation isshowninFigure1.1.TheideaofOFDMistodistributethehighdatastreamtomanylowratedata streamsthataretransmitted inaparallelwayovermanysubchannelswithin thesamebandwidth[4].Thusineach subchannelthesymbol durationislowascomparedtothemaximum delayofthechannelandnowISIcanbe handled[5].

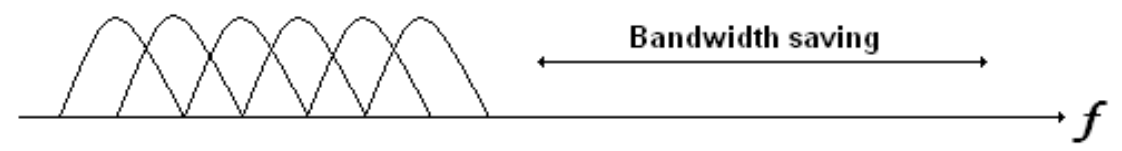

Figure 1.1:OrthogonalFrequencyDivisionMultiplexing(OFDM)multicarriermodulation

Inordertoeliminate further the effect ofICIandISI,OFDM introduces whatiscalledtheCyclicPrefix (CP), whichisaguardintervalbetweentwoconsecutiveOFDMsymbols[6].TheCPismade bycopyingthelast partoftime domainOFDMsymbolandappendingittothebeginningoftheOFDM symbolintheguardinterval, itisa cyclicextensionoftheOFDMsymbol[7].TheprocedureisshowninFigure1.2. 


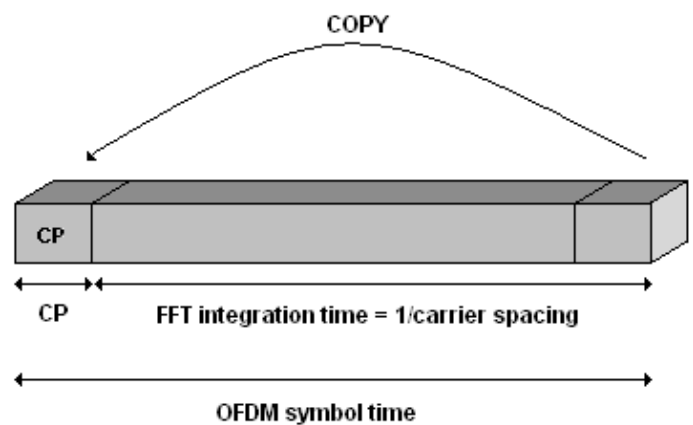

Figure 1.2:OFDMSymbolwithCyclicPrefix

InasingleOFDMtransmission

allthesubcarriersaresynchronizedtoeachother,restrictingthe transmissiontodigitalmodulation schemes.OFDMissymbolbased,andcanbethoughtofasalargenumberof low bitratecarrierstransmittinginparallel.SincethesemultiplecarriersformasingleOFDMtransmission,they arecommonlyreferredtoas subcarriers, withthetermofcarrierreservedfor describingtheRFcarriermixingthe signal frombaseband[8].TheoverallOFDMmodelisgiveninFigure 1.3

Data in
Data out
Data Encoder
Data Deco der
QAM IFFT
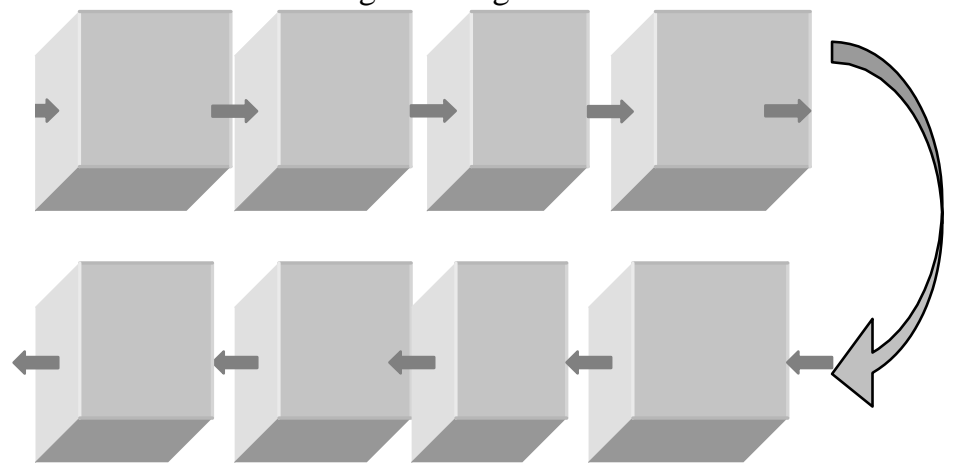

\section{QAD \\ Cyclic \\ Prefix \\ Cyclic Prefix Remov \\ FFT}

Figure 1.3:OFDMModel

\section{Methodology}

Inmultimediacommunication,ademandemergesforhigh-speed,high-quality digitalmobileportable receptionandtransmission. Areceiverhastocopewitha signalthat is oftenweakerthandesirableandthat contains manyechoes.Simpledigitalsystemsdo notworkwellinthemultipathenvironment.

Foragivenoveralldatarate,increasing thenumberofcarriersreducesthedataratethateachindividual carriermustconvey,andhence lengthensthesymbolperiod.Thismeansthattheinter-symbol interferenceaffectsa smallerpercentageofeachsymbolasthenumberofcarriersandhencethesymbolperiodincreases. Asinglecarrier system,theresponsesofindividual bitsareoverlapping,thuscreatingISI.Inaconventional serialdatasystem,the symbolsaretransmittedsequentially, with thefrequencyspectrumofeach datasymbolallowed tooccupytheentire availablebandwidth.Inaparalleldatatransmission systemseveralsymbolsaretransmittedatthesametime, what offerspossibilities foralleviatingmanyoftheproblemsencounteredwithserialsystems.InOFDM,thedatais dividedamonglargenumberofcloselyspacedcarriers. Theentirebandwidth isfilledfromasinglesourceofdata. Insteadoftransmitting inserialway,dataistransferredinaparallelway[5].Onlyasmallamountofthedatais carriedoneachcarrierandbythisloweringofthebitratepercarrier(notthetotalbitrate), theinfluenceofinter-

symbolinterferenceissignificantly reduced.AnimportantpartoftheOFDMsystemdesignthatthebandwidth occupiedisgreaterthanthecorrelationband widthofthefadingchannel.Althoughsomeofthecarriersare degraded bymultipathfading,themajorityofthecarriersshouldstillbeadequatelyreceived[9].

Itispossible,however,toarrange thecarriersinanOFDMsignalsothatthesidebandsoftheindividual carriersoverlapandthesignalscanstillbe receivedwithoutadjacentcarrierinterference[10].Inorder todothisthe carriersmustbemathematicallyorthogonal[8]. The receiveractsas abankofdemodulators,translatingeachcarrier downtoDC,theresulting signalthenbeingintegrated overasymbol periodtorecovertheraw data.Iftheother carriersallbeatdowntofrequencieswhich,inthetimedomain, haveawholenumberofcyclesinthesymbol 
period,thentheintegrationprocess results inzerocontributionfrom allthesecarriers. Thusthecarriersarelinearly independent(i.e.orthogonal).

Mathematically,supposewe havea setofsignals $\psi$,

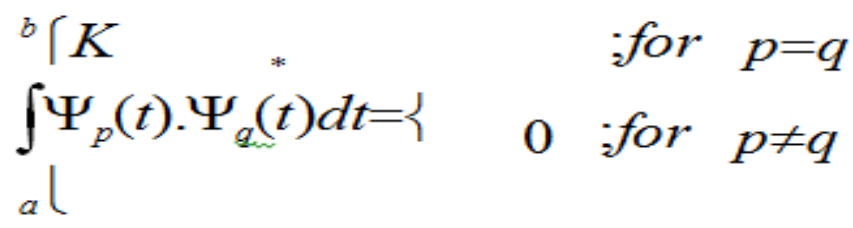

Wherethe*indicatesthecomplexconjugate andinterval[a,b]isasymbol period.Afairlysimplemathematical proofexists, thattheseriessine $(\mathrm{mx})$ form $=1,2, \ldots$ isorthogonalovertheinterval.

Inordertoavoidalargenumberofmodulatorsandfiltersatthetransmitterandcomplementary filtersand demodulatorsatthereceiver, itisdesirabletobeabletousemoderndigitalsignalprocessingtechniques, suchas fastFouriertransform(FFT).Mathematically,eachcarriercanbedescribedasa complexwave.

$$
s_{c}(t)=A(t) \cdot e^{j\left[\omega_{c} t+\phi_{c}(t)\right]}
$$

Thevaluesoftheparametersareconstantoverthesymboldurationperiod.OFDMconsistsofmanycarriers. Thus thecomplexsignals $S_{S}(t)$ arerepresentedby:

$$
s(t){ }_{{ }^{s} N}^{\stackrel{1}{\underline{1}}} \sum_{n=0}^{N-1} A_{N}(t) \cdot e^{j\left[\omega_{n} t+\phi_{x L}(t)\right]}
$$

\section{Where}

$$
\omega_{n}=\omega_{0}+n \Delta \omega
$$

Thisisofcourseacontinuous signal.Ifweconsiderthewaveforms ofeachcomponent ofthesignaloverone symbolperiod,thenthevariablestakeonfixedvalues, whichdependonthefrequency ofthatparticularcarrier, andsocanberewritten:

$$
\begin{aligned}
& \phi_{n}(t)=\phi_{n} \\
& A_{n}(t)=A_{n}
\end{aligned}
$$

Ifthesignalissampledusinga samplingfrequencyof1/T,thentheresultingsignalisrepresentedby:

$$
s(k T)={ }^{s} N \sum_{n=0}^{N-1} A e^{j\left[\left(\omega_{0}+n \Delta \omega\right) k T+\phi_{n}\right]}
$$

\section{$\tau=N T$}

If we nowsimplifyeq.5.3, withoutalossofgenerality,thenthe signalbecomes:

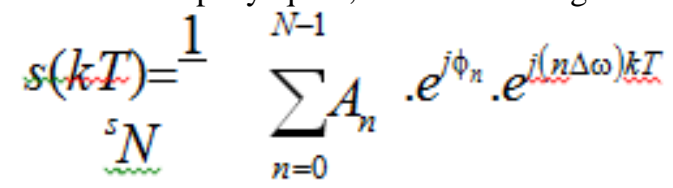

NowEq.5.4canbecomparedwiththe generalformoftheinverseFouriertransform: 


$$
g(k T)=^{1} \sum_{N_{n=0}}^{N-1}\left(\frac{n}{(N T)} \mid \cdot e^{i \frac{2 \pi n k}{N}}\right.
$$

Ineq.5.4,thefunction ${ }^{A . e^{j \phi_{*}}}$ isnomorethanadefinitionofthesignal in thesampledfrequencydomain,and $\mathrm{s}(\mathrm{kT})$ isthetimedomainrepresentation.Eqs.5.4and5.5areequivalentif:

$$
\Delta f=\frac{\Delta \omega}{2 \pi}=\frac{1}{N T}=\underline{1}
$$

This is the same condition that was required for orthogonality. Thus, oneconsequence of maintaining orthogonalityisthattheOFDMsignalcanbedefinedbyusingFouriertransformprocedures.

\subsection{TheuseoftheFFTinOFDM}

Atthetransmitter, thesignalis

definedinthefrequencydomain.Itisasampleddigitalsignal,and itisdefinedsuchthatthediscreteFourierspectrum existsonlyatdiscretefrequencies. EachOFDMcarrier correspondstoone elementofthisdiscreteFourierspectrum.The amplitudesand phasesofthecarriersdependon thedatatobetransmitted. Thedatatransitions aresynchronized atthecarriers,andcanbeprocessedtogether, symbolbysymbol[11].Thedefinitionofthe(N-point)discreteFouriertransform(DFT)is:

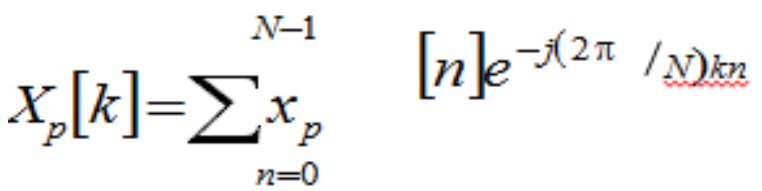

Andthe(N-point)inversediscreteFouriertransforms(IDFT):

$$
\underset{{ }_{n}}{x[n]}=\stackrel{1}{N-1} \sum_{k=0} X_{e}[k] e^{-j(2 \pi / N) k n}
$$

Anaturalconsequenceofthismethod isthatitallowsustogeneratecarriersthatareorthogonal.Themembersof anorthogonalsetarelinearlyindependent.Consideradatasequence $\left(\mathrm{d}_{0}, \mathrm{~d}_{1}, \mathrm{~d}_{2}, \ldots, \mathrm{d} \mathrm{N}-1\right)$, whereeachdnisa complexnumberd $_{n}=a_{n}+j b_{n}$

$$
D_{m}=\sum_{n=0}^{N-1} d_{n} \cdot e^{-j(2 \pi n m \mathcal{K})}=\sum_{n=0}^{N-1} d{ }_{n} \cdot e^{-2 j \pi f_{n} t_{m}}
$$

Therealpartofthe vectorD hascomponents;

$$
Y_{m}=\operatorname{Re}\left\{D_{m}\right\}=\sum_{n=0}^{N-1}\left[a_{n} \cos \left(2 \pi f_{n} t_{m}\right)+b_{n} \sin \left(2 \pi f_{n} t_{m}\right)\right] \quad ; k=0,1,2, \ldots, N-1
$$

Ifthesecomponentsareappliedtoalow-passfilterattimeintervals, asignalisobtainedthatclosely approximatesthefrequencydivisionmultiplexedsignal

$$
y(t)=\sum_{n=0}^{N-1}\left[a_{n} \cos \left(2 \pi f_{n} t_{m}\right)+b_{n} \sin \left(2 \pi f_{n} t_{m}\right)\right] \quad ; 0 \leq t \leq N \Delta t
$$


ISItakesplacewhenechoesondifferent-lengthpropagationpathsresultinoverlappingreceivedsymbols [12].ProblemscanoccurwhenoneOFDMsymboloverlapswiththenextone.Thereisnocorrelationbetween twoconsecutive

OFDMsymbolsandthereforeinterferencefromonesymbolwiththeotherwillresultina disturbedsignal[13].

Inaddition,oncetheincomingsignalissplitintotherespectivetransmissionsub-carriers,aguardintervalis added

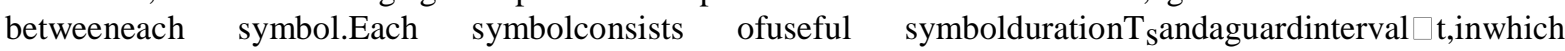
asignalof $\mathrm{T}_{\mathrm{S}}$ iscyclicallyrepeated.ThisisshowninFigure1.4.

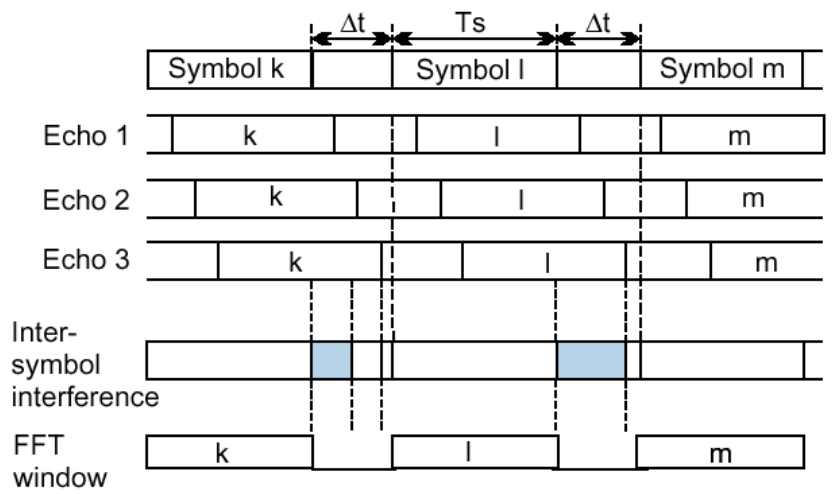

Figure 1.4:CombatingISI usingaguardinterval

\subsection{OFDMGenerationand Reception}

OFDMsignalsaretypicallygenerateddigitallyduetothedifficultyincreatinglargebanksof phaselockoscillatorsand receiversintheanalogdomain.Figure 1.5showsthe blockdiagramofatypical OFDM transceiver.Thetransmittersectionconvertsdigital datatobetransmitted,intoamappingofsubcarrieramplitude andphase.ItthentransformsthisspectralrepresentationofthedataintothetimedomainusinganInverse

DiscreteFourierTransform (IDFT).TheInverseFastFourierTransform (IFFT)performsthesameoperationsas anIDFT,exceptthatitismuchmorecomputationallyefficiency,andsoisusedinallpracticalsystems[11].In order totransmittheOFDMsignalthecalculatedtimedomainsignalisthen mixeduptotherequiredfrequency.

Thereceiverperformsthereverseoperation ofthetransmitter, mixingtheRFsignaltobasebandfor processing,thenusingaFastFourierTransform (FFT)toanalyzethesignalinthefrequencydomain.The amplitudeandphaseofthesubcarriersarethenpickedoutand convertedbacktodigitaldata.

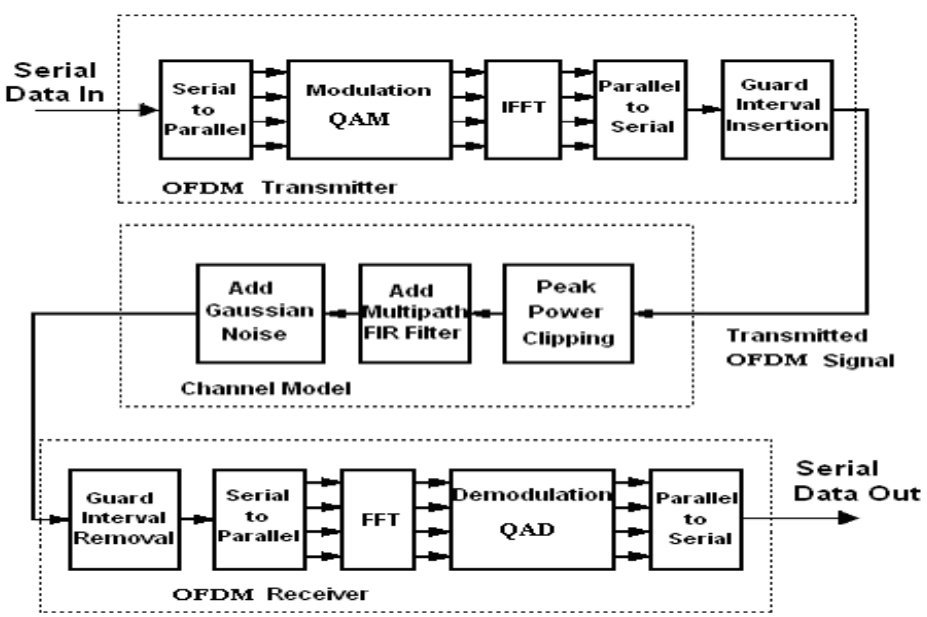

Figure 1.5:CompleteOFDMModel,withchannelcharacteristics

\subsection{Serialto ParallelConversion}

Datatobetransmittedistypically intheform ofaserialdatastream.InOFDM,eachsymboltypically transmits40- 4000 bits, andsoaserialto parallelconversionstageisneededtoconverttheinputserialbitstream tothedatatobetransmittedineachOFDMsymbol.Thedataallocatedtoeachsymboldependsonthemodulation scheme used andthenumber ofsubcarriers.For asubcarriermodulationof16-QAMeachsubcarriercarries4bits ofdata,andsofora transmissionusing100subcarriersthenumberofbitspersymbolwouldbe 400[14]. 


\subsection{SubcarrierModulationMapping}

TheOFDMsubcarrierscanbemodulated byusing16-QAM[15].Theconversion canbeperformed accordingtothegraycodeconstellationmappings, withtheinputbitb $b_{O} \quad$ beingtheearliestinthestream.The outputvaluesdareformedbymultiplyingtheresultingvalue $(\mathrm{I}+\mathrm{jQ})$ byanormalizationfactorK $\mathrm{MOD}$ asdescribedas $d \square \square I \square j Q \square \square K_{M O D}$. ThenormalizationfactorKMOD

for16-QAMis1/ $110 . I n 16-$

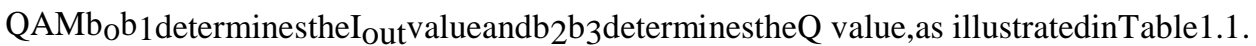

Table1.1:16-QAMencodingTable.

\begin{tabular}{|l|l|l|l|}
\hline Inputbits $\left(\mathrm{b}_{0} \mathrm{~b}_{1}\right)$ & I-out & Inputbits( $\left.\mathrm{b}_{2} \mathrm{~b}_{3}\right)$ & Q-out \\
\hline 00 & -3 & 00 & -3 \\
\hline 01 & -1 & 01 & -1 \\
\hline 11 & 1 & 11 & 1 \\
\hline 10 & 3 & 10 & 3 \\
\hline
\end{tabular}

Onceeachsubcarrierhasbeenallocatedbitsfortransmission,theyaremappedusingamodulation schemetoasubcarrieramplitude andphase,whichisrepresented byacomplex In-phaseandQuadrature-phase (IQ)vector.Figure1.6showsanexampleofsubcarriermodulationmapping.Thisexampleshows16-QAM， which maps4 bitsforeachsymbol.

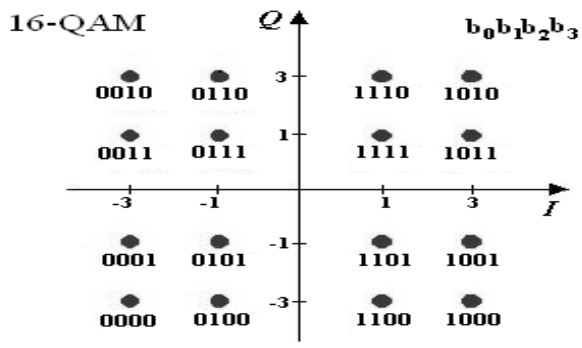

Inthereceiver,mappingthe receivedIQvector back tothedatawordperforms subcarrierdemodulation. Duringtransmission,noiseanddistortionbecomesaddedtothesignalduetothermalnoise,signalpower reduction andimperfectchannelequalization.Figure1.7showsanexampleofareceived16-QAMsignalwitha SNRof18dB.EachoftheIQpointsisblurredinlocationduetothechannelnoise.ForeachreceivedIQvector the receiverhas to estimate the most likelyoriginal transmissionvector. This isachievedby findingthe transmission vectorthatisclosesttothereceivedvector.Errorsoccurwhenthenoiseexceedshalfthespacing betweenthetransmissionIQpoints, makingitcrossovera decisionboundary.

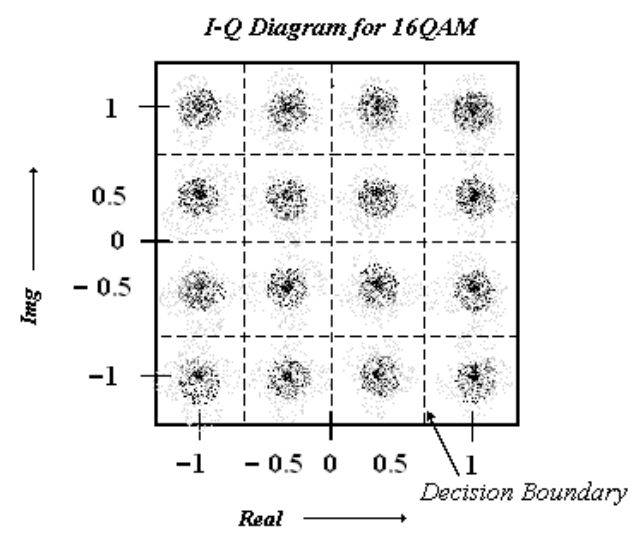

Figure1.7:IQplotfor 16-QAMdata withaddednoise.

\subsection{FrequencytoTimeDomainConversion}

Afterthesubcarriermodulationstageeachofthedatasubcarriersissettoanamplitudeandphase 
tobetransmitted[16].Figure1.8showstheIFFTsectionoftheOFDMtransmitter.Inthefrequency domain, beforeapplying theIFFT,eachofthediscretesamples oftheIFFTcorrespondstoanindividual subcarrier.Most ofthesubcarriers aremodulatedwithdata.Theoutersubcarriers areun-modulated andsettozeroamplitude. Thesezerosubcarriersprovideafrequency guardbandbeforetheNyquistfrequencyandeffectively actasan interpolationofthe signalandallowsfora realisticrolloffintheanaloganti-aliasingreconstructionfilters.

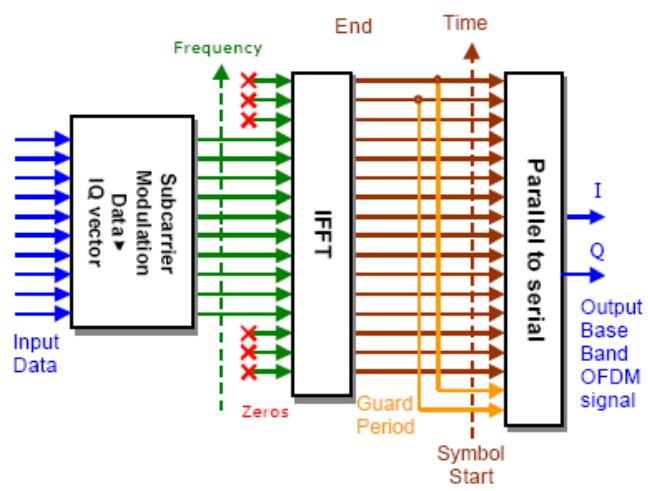

\section{Simulation results}

Dataencoderproduces binarycodesforanyapplied signalthatiswecansaythatitperformsAnalog to Digitalconversion.Asperformedthedigital processing,forthiswerequiredigital datatobeapplied.Theapplied datawas notindigital format sodata encoderisusedforconvertingnon-digitaldata to digital form. The encoders usedaredifferent forallthreetypes ofinputdata.Theencoder outforTextandimage isshowninFigure 1.9and 1.10respectively.

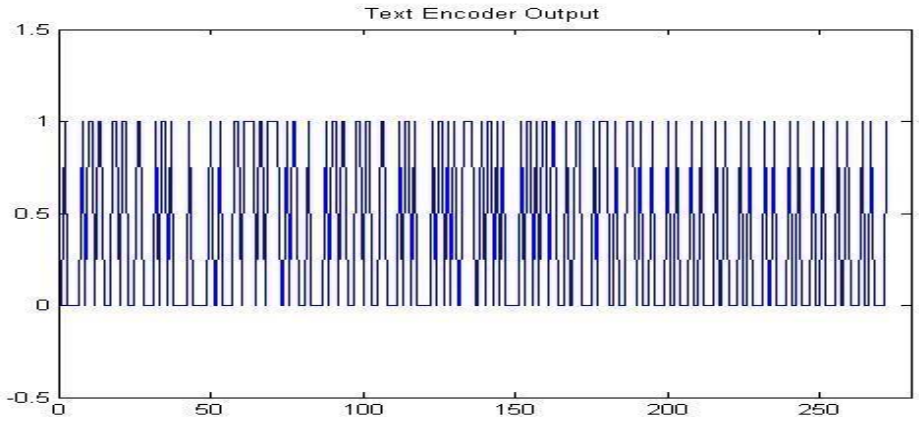

Figure 1.9:TextEncoderOutput

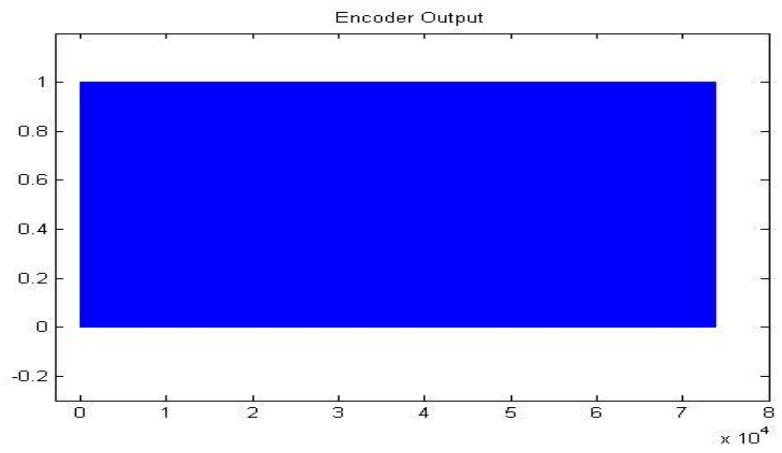

Figure1.10:ImageEncoderOutput

\subsection{SubCarrierModulation(QAM)}

Onceeachsubcarrierhasbeenallocatedbitsfortransmission,

theyaremappedusingamodulation schemetoasubcarrieramplitude andphase,whichisrepresented byacomplexIn-phaseandQuadrature-phase (IQ)vector.Figure1.11showsanexample ofsubcarrier modulationmapping.Thisexampleshows16-QAM, which maps4 bitsforeachsymbol. 
Inthereceiver,mappingthereceivedIQvectorbacktothedatawordperforms subcarrier demodulation.During transmission, noiseanddistortionbecomes addedtothesignalduetothermalnoise,signal power reductionandimperfectchannelequalization.Figure1.12showsanexampleofareceived16-QAMsignal withaSNRof18dB.EachoftheIQpointsisblurredinlocation duetothechannelnoise.ForeachreceivedIQ vectorthereceiverhastoestimatethemostlikelyoriginaltransmissionvector.Thisisachievedbyfindingthe transmissionvectorthatisclosesttothereceivedvector.Errorsoccurwhenthenoiseexceedshalfthespacing betweenthetransmission IQpoints,makingitcrossoveradecisionboundary.ThesimulatedresultofQAMis showninFigure1.13.

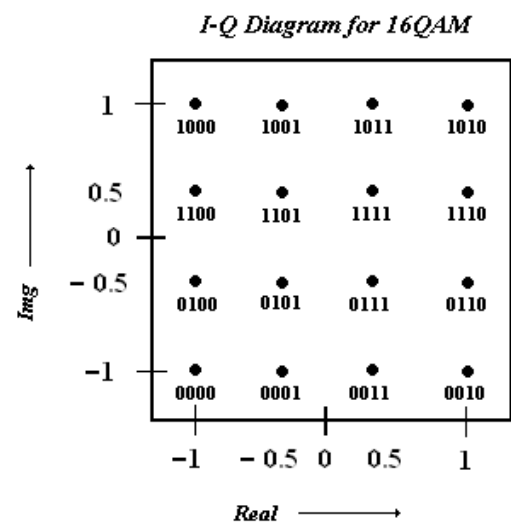

Figure1.11:ExampleIQmodulationconstellation(16-QAM,withgraycodingofthedatatoeachlocation)

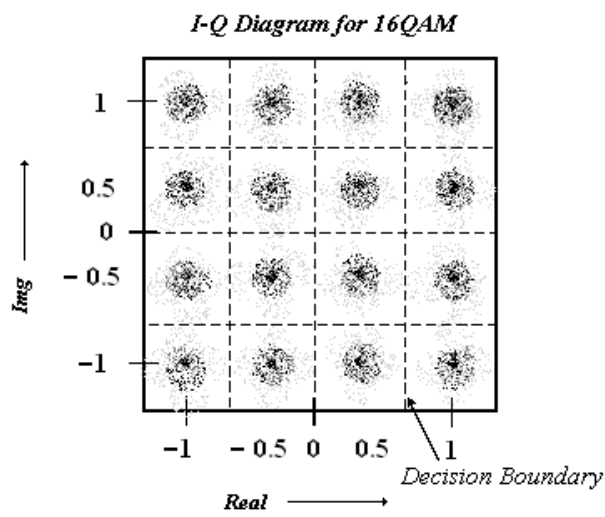

Figure1.12:IQplotfor16-QAMdata withaddednoise.

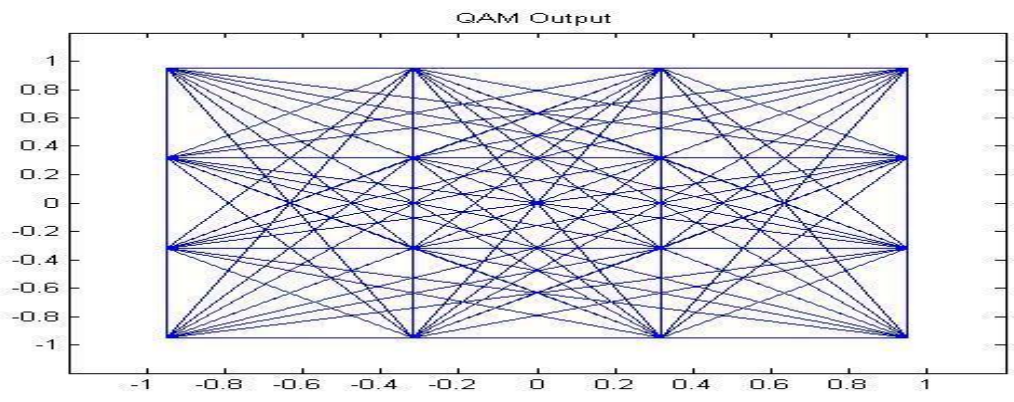

Figure1.13:16-QAMOutput

\subsection{InverseFastFourierTransform(IFFT)}

InthisstagetheInverseFastFourierTransformtakesplace.Thesignalistransformedfromfrequency domaintoitstimedomainanddifferentorthogonalsubcarriers aresetup.Itisthemostimportantstagein theOFDMtransmitter; itisthestagewhichgivesustheconceptoforthogonality.TheIFFTfortextand imageare showninFigure1.14andFigure1.15respectively. 


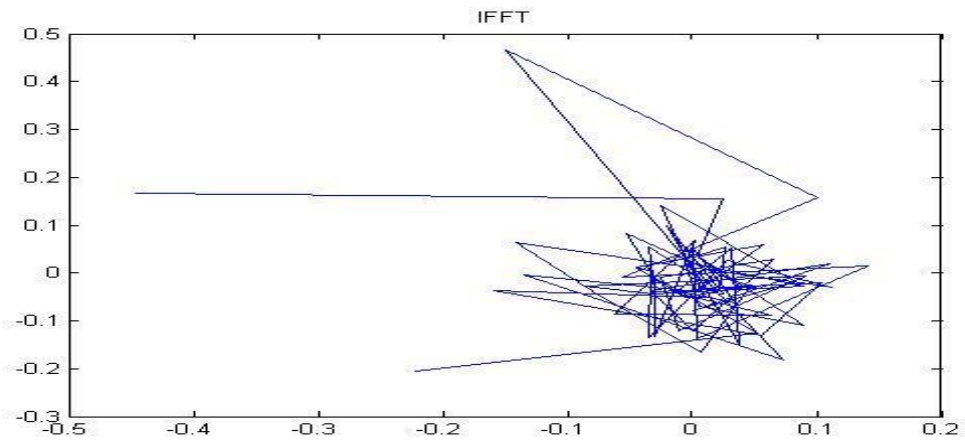

Figure1.14:IFFTfortextdata

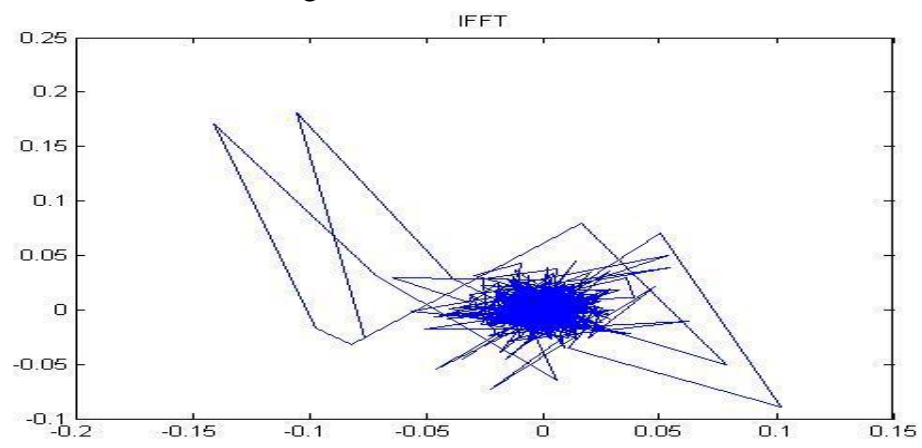

Figure1.15:IFFTforimageData

\subsection{FastFourierTransform(FFT)}

Atthereceivingendthesignalisagaintransformedfromtimedomain tofrequencydomain.TheFFT fortextandimageare showninFigure1.16and1.17accordingly.

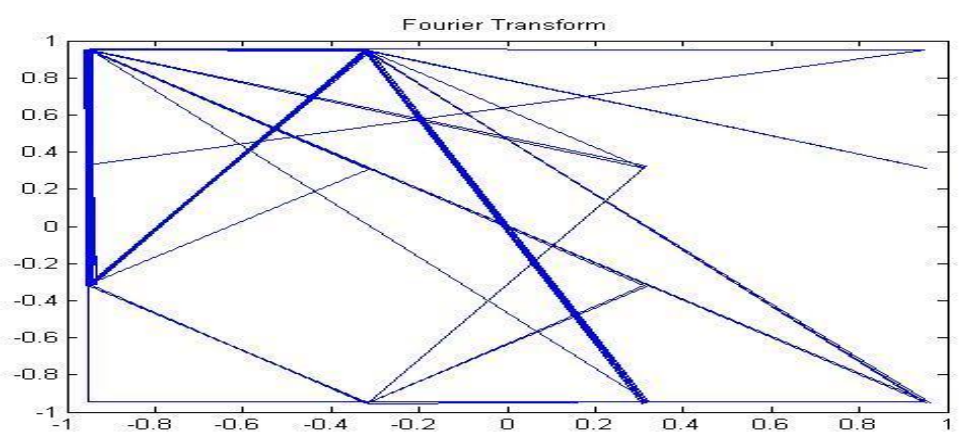

Figure1.16:FFT fortextdata

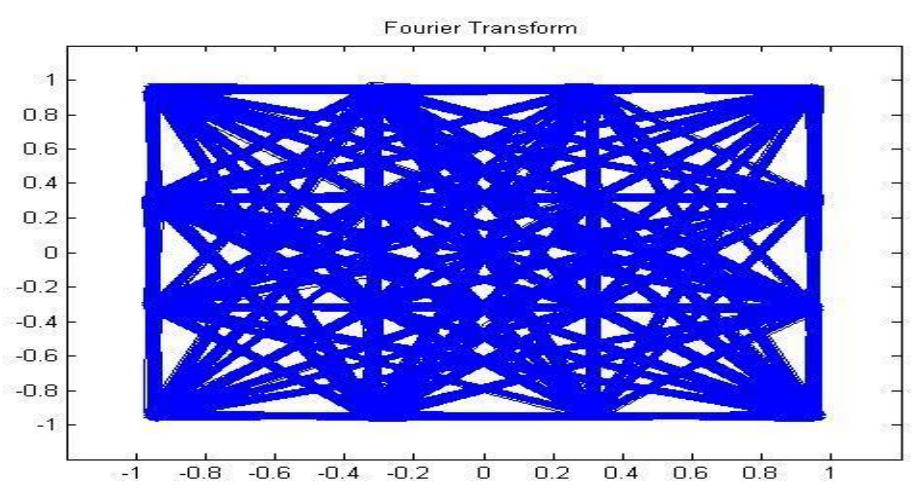

Figure1.17:FFT forImagedata 


\subsection{Subcarrierdemodulation(QAD)}

QuadratureAmplitudeDemodulation(QAD)isusedtodemodulatethesignalfromsubcarriers.For thisweuse.ItistheexactreverseofQAMasshowninFigure1.18and1.19respectively.

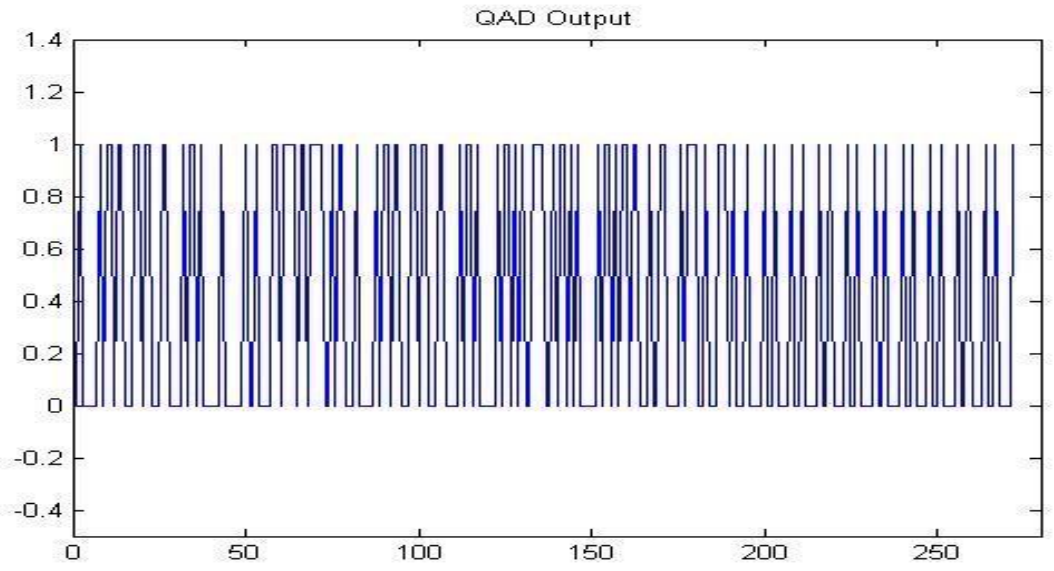

Figure1.18:QADdemodulationforTextdata

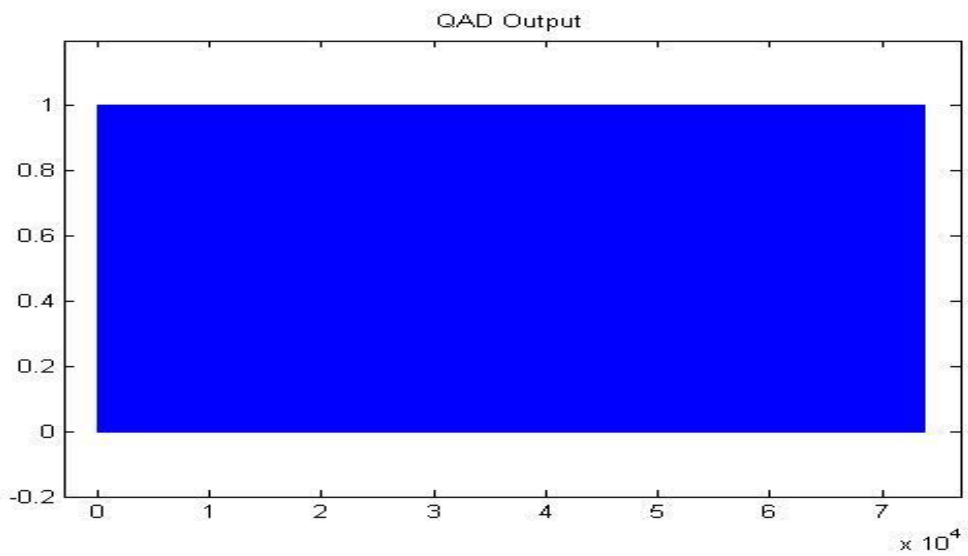

Figure1.19:QADdemodulationforImagedata

\subsection{DataDecoder}

Thedemodulatedsignalisthenappliedtothedataencoder,whichconvertsthebinaryencodeddata intonormalform.ThisisourrequiredoutputwhichisshowninFigure1.20.

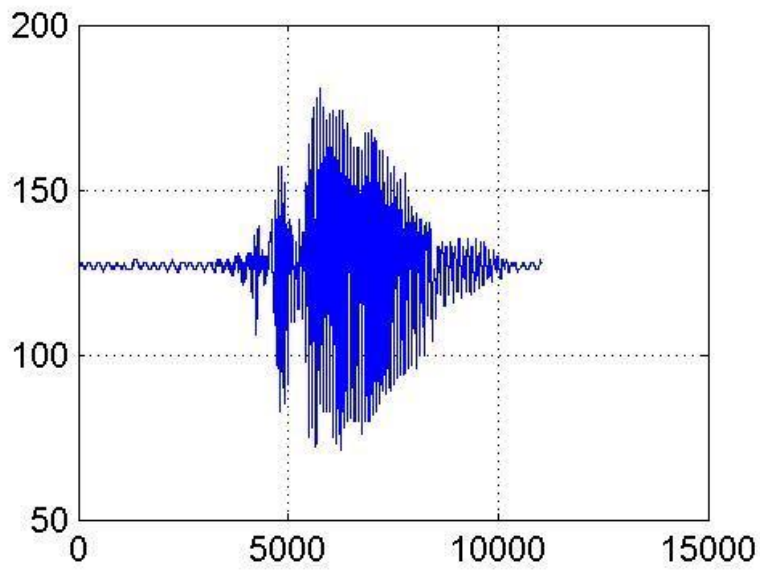

(a)

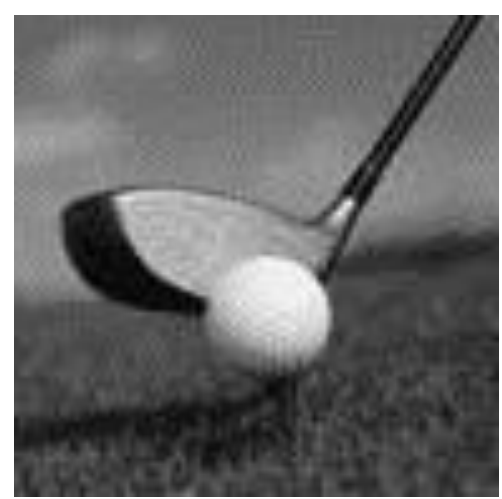

(b) (c)AllahistheSupremePower.

Figure 1.20:Receivedinformation,(a)AudioSignal,(b)Image,(c)Text 
3.6CyclicPrefix

AllthecyclicprefixandcyclicprefixremovalfortextandimageareshowninFigure 1.21

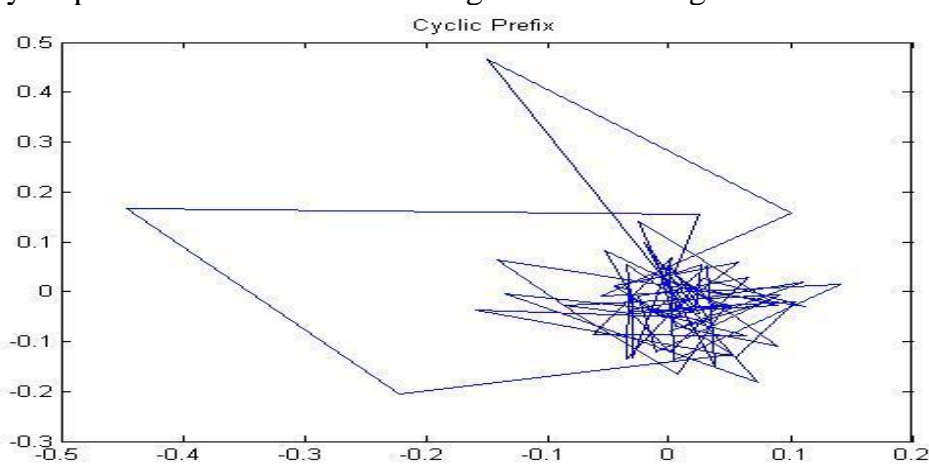

(a)

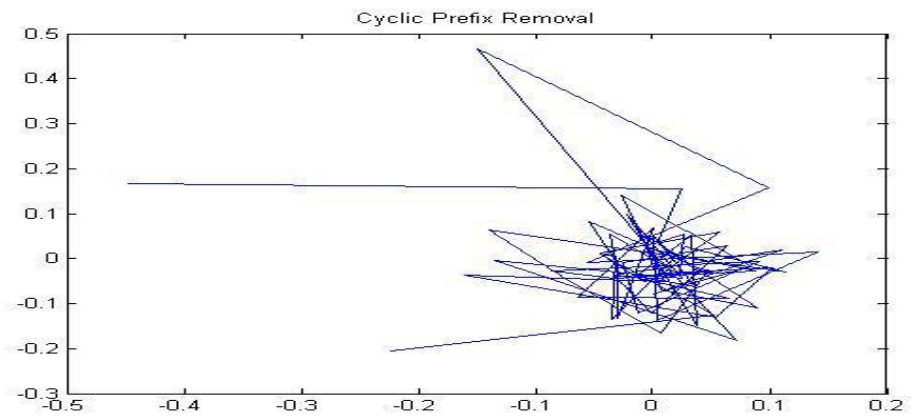

(b)

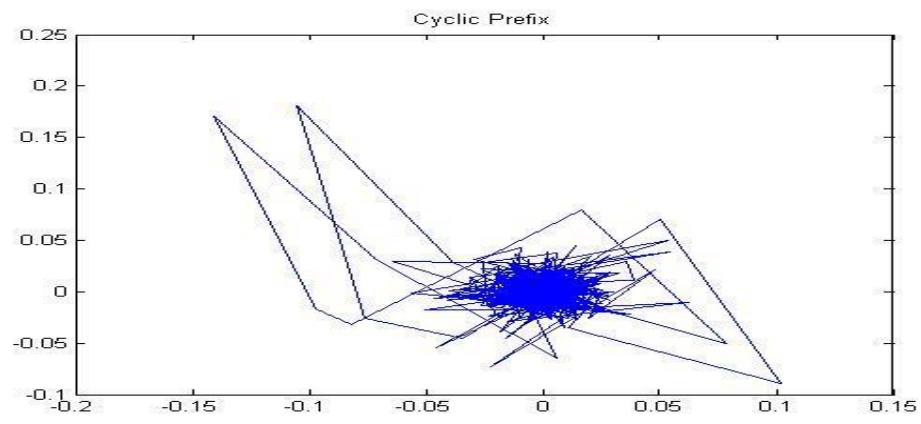

(C)

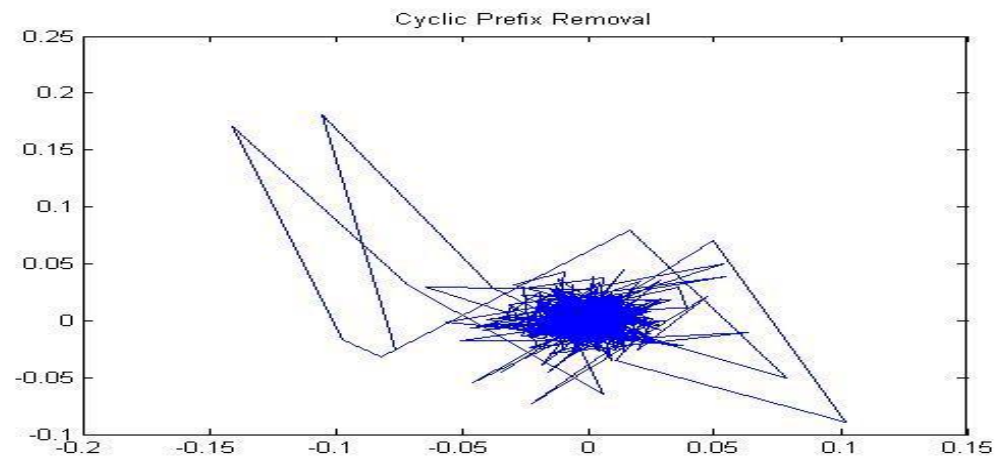

(d)

Figure 1.21:(a)cyclicprefixfortextdata(b)cyclicprefixremovalfortextdata (C)Cyclicprefixforimagedata(d) CyclicprefixremovalforImagedata 


\section{Conclusion}

The purposeofthisworkistogive poweroftheOFDMtransmissionscheme.Herenotonly butalsosomeoftheproblems thatwerepresentincommunicationas wellasthetechniquestocorrectthem.

Thismodelfulfillsalmostallthebasicbuilding blocksofacommercial OFDMsystemforboththetransmitting sideandthereceivingsidesoperationsusingMATLAB.WeperformedarealtimesimulationofOFDMmodel, byapplyingthreedifferent typesofdata,whichareText,ImageandAudio.Textwasfeddirectly throughkey boardandwasstoredinacharactertypedataarray.Wegotdifferent imagesfrom systemmemoryandappliedto ourmodel.Themodel issupportingonly gray scaledimages,forthisweconvertedtheRGBimage tograyscaled image.We got audiodata fromexternalsideusinga microphone. OFDMprove torevolutionizemobile communications byallowingittobemorereliableandrobustwhilemaintainingthehighdataratethatdigital communication demands.Toachievehighspectrum efficiency,parameterssuchascyclicprefix,numberof carriers,andconstellationmustadapttothecurrentsituationofthecommunicationchannel.Hereitisshownthat suchflexibilitycanbeobtainedwithareasonableamountofextrahardware.Theflexibility alsocontributestoa largersetofpossibleapplications andthuslargerfabricationvolumesandlowerprice.Theonlyextrasourceof powerconsumptionduetotheflexibilityistheleakagepowerintheunusedpartsofthedesign.TheOFDM modelhavebeenimplemented bysimulation andimplementedinrealtimeoperationbyusingDigitalSignal processorKit(DSK).Thesimulation onOFDMmodel canbeextendedbyintroducing multipatheffectsand channel'sandsystem'snonlinearitieswhichmakestheOFDM simulationa fullfunctionalpractice.

\section{References}

[1] HuilinXu, Chia-Chin Chong, Guvenc I, Watanabe F., "High-Resolution TOA Estimation with Multi-Band OFDM UWB Signals," IEEE Int. Conference, Beijing, pp-4191-4196, May, 2008.

[2] Batra, A. Balakrishnan, J. Dabak, A., "Multi-band OFDM: a new approach for UWB," DSPS R\&D Center, Texas Instrum., Dallas, TX, USA, Circuits and System,2004. ISCAS'04, Proceedings of the 2004 International Symposium, Volume: 5, pp- 365-368.

[3] Michal Litwin, "Orthogonal Frequency Division Multiplexing (OFDM) Transceiver Design," Master Thesis, Dept. of Electrical Engineering Program, Cornell University, August, 2010.

[4] Guillermo Acosta, “OFDM Simulation Using Matlab," Smart Antenna Research Laboratory, August, 2000

[5] P. G. Lin, "OFDM Simulation in MATLAB," Dept. of Electrical Engineering, California Polytechnic State University, San Luis Obispo, June, 2010.

[6] Shaoping Chen, "ICI and ISI analysis and mitigation for OFDM systems with insufficient cyclic prefix in time-varying channels, "IEEE Consumer Electronics Society, Dept. of Electron. \& Information Eng., South-Central Nat. Univ., China, Feb. 2004

[7] Magnus, S. J.J Van de Beek, P. O. Borjesson, "Timing and Frequency Synchronization in OFDM System using Cyclic Prefix", Div. of Signal Processing, Lulea University of Technology S-971-87 Lulea, Sweden.

[8] Charan Langton, "Orthogonal Frequency Division Multiplexing (OFDM)", Intuitive Guide to Principles of Communication, pp. 122, 2004.

[9] Islam, Mohammed Safiqul, BaraiGouri Rani, Mahmood, Atiq. "Performance analysis of different modulation schemes using OFDM techniques in Rayleigh fading channel, ” International Journal of Fundamental Physical Sciences, Mar. 2011, Vol. 1 Issue 1, p22-27.6p.

[10] Y. Chiu, D. Markovi, H. Tang, and N. Zhang, "OFDM Receiver Design," 2000

[11] J. Kuo, C. Lin, and A. A. Wu, "VLSI Design of a Variable-Length FFT / IFFT Processor for OFDM-Based Communication Systems, ”EURASIPJournalonAppliedSignalProcessing, 1306-1316c, pp. 1306-1316, 2003

[12] Zhi-QuanLuo ; Davidson, T.N. ; Giannakis, G.B. ; Wong, Kon Max, "Transceiver optimization for block-based multiple access through ISI channels”, IEEE Tran. Vol. 52, Issue: 4, pp- 1037 - 1052 April, 2004

[13] Le Van Ninh, Trinh Anh Vu, Huu Tue Huynh, Fortier, P. "New Cyclic Prefix Based Symbol Timing and Carrier Synchronization for OFDM." Communications, 2006 23rd Biennial Symposium pp-198 - 203, 2006

[14] Haithamj.H., M. F. M. Salleh, "Performance Analysis of QAM-Modulation Parameters on FFT-OFDM System over Doppler Frequency Fading Channels," Int. Journal of Info. and Comm. Tech., Vol. 3, Issue 1, pp-40-52, April 2011

[15] Y. Shao, N. Chi, J. Fan, and W. Fang, "Generation of 16-QAM-OFDM Signals Using Selected Mapping Method and Its Application in Optical Millimeter-Wave Access System,” IEEE Photonics Technology Letters, vol. 24, no. 15, pp. 1301-1303, 2012.

[16] Cortes, A. Velez, I. Sevillano, J.F. Irizar, A. "An approach to simplify the design of IFFT/FFT cores for OFDM systems", IEEE Transactions, Vol. 52 Issue 1, pp-26-32, Feb.2006 